\title{
ESTUDOS DE CASO SOBRE CUSTOS \\ AMBIENTAIS: ÊNFASE NOS PROCEDIMENTOS METODOLÓGICOS
}

\section{CASE STUDY ABOUT ENVIRONMENTAL COSTS: EMPHASIS ON THE METHODOLOGIES PROCEDURES}




\section{RESUMO}

A estratégia de pesquisa estudo de caso, embora muito criticada, é escolhida por um grande número de pesquisadores, com teses, dissertações e artigos publicados sobre diferentes aspectos nas ciências sociais. As investigações sobre custos ambientais têm demonstrado áreas que estão em contínuo desenvolvimento, como a mensuração, necessidade de registro, controle e gerenciamento, por exemplo. $\mathrm{O}$ objetivo deste artigo é analisar os estudos de caso que abordaram a identificação e mensuração dos custos ambientais. Trata-se de uma pesquisa descritiva, e, quanto aos procedimentos metodológicos, utilizaram-se a pesquisa bibliográfica e análise de conteúdo das contribuições acadêmicas de 34 teses e dissertações defendidas em programas de pós-graduação reconhecidos e credenciados pela Capes. Os resultados da pesquisa revelaram que os procedimentos metodológicos mais utilizados para coleta de dados foram entrevistas, pesquisa documental e aplicação de questionário. A maior parte dos trabalhos analisados não estabeleceu uma relação entre os resultados obtidos com o referencial teórico citado.

\section{PALAVRAS-CHAVE}

Estudo de caso; Custos ambientais; Estratégia de pesquisa; Metodologia; Análise de conteúdo.

\section{ABSTRACT}

The research strategy case study, even so very criticized, is chosen by a great number of researchers, with thesis, dissertations and articles published on different aspects in social sciences. The inquiries on ambient costs have demonstrated areas that are in continuous development, as the measurement, necessity of register, have controlled and management, for example. The objective of this article is to analyze the case studies that had approached the identification and measurement of the environmental costs. The Methodologies procedures used in this descriptive 
study were the bibliographical research, documentary and content analysis of the academic contributions, that is, 34 thesis and dissertations defended in recognized and credential programs of after-graduation for the Capes. It was founded that the used methodologies procedures more for collection of data had been interviews, documentary research and application of questionnaire. Most of the analyzed works did not establish a relation between the results gotten with the theoretical cited.

\section{KEYWORDS}

Case study; Environmental costs; Strategy of research; Methodology; Content analysis.

\section{INTRODUÇÃO}

É perceptível o considerável aumento da produção acadêmica nas últimas décadas, e, tendo em vista a aprovação de um maior número de cursos de graduação e pós-graduação oferecidos no país, é natural o aumento quantitativo de estudantes interessados no aprofundamento em pesquisas e no aperfeiçoamento destas.

Assim, observa-se a grande diversidade da produção bibliográfica sobre todas as áreas do conhecimento científico. Os avanços tecnológicos têm proporcionado a facilidade de acesso a tais produções, possibilitando discussões abrangentes, como também a avaliação qualitativa e quantitativa dos trabalhos que são disponibilizados.

A pesquisa acadêmica com foco na questão ambiental tem ganhado cada vez mais espaço em praticamente todas as áreas. As discussões sobre metodologia da pesquisa aplicada nas ciências sociais são intensas, o que permite o avanço do conhecimento proporcionado pelas diferentes ideias e pelos pontos de vista que são debatidos.

A estratégia de pesquisa estudo de caso, embora muito criticada, apresenta-se como preferencial para um grande número de acadêmicos, com artigos, dissertações e teses publicados sobre diferentes aspectos relacionados à questão ambiental.

As pesquisas sobre custos ambientais têm focalizado áreas complexas que estão em contínuo desenvolvimento, como a mensuração, necessidade de registro, o controle e gerenciamento desses custos. Dessa forma, o problema que se 
formula neste artigo é a contribuição das pesquisas anteriores com ênfase nos custos ambientais e baseadas na estratégia estudo de caso. O objetivo deste artigo é analisar os estudos de caso que abordaram a identificação e mensuração de custos ambientais.

Trata-se de um estudo descritivo, e, quanto aos procedimentos metodológicos, a pesquisa bibliográfica foi utilizada na verificação da produção acadêmica sobre o tema, com base no exame dos trabalhos de eminentes autores que se dedicaram a estudar o assunto. A análise de conteúdo, com base em contribuições que abordaram os custos ambientais, complementou a pesquisa.

A principal motivação para esta investigação foi a percepção de quem se propõe a elaborá-la; que na sua experiência acadêmica, vislumbrou-se com as deficiências metodológicas dos estudos de caso de sua própria autoria.

Tendo em vista que, há mais de uma década, o acúmulo de conhecimento sobre custos ambientais é significativo, o que pode ser inferido a respeito das contribuições acadêmicas quanto aos aspectos metodológicos?

Considera-se que este artigo poderá contribuir para discussões acerca da elaboração de estudos de caso, assim como para o desenvolvimento teórico sobre custos ambientais.

Algumas delimitações devem ser destacadas: diferentemente de várias pesquisas que recentemente têm apresentado levantamentos quantitativos sobre a produção em anais de congressos de determinados períodos sobre alguma temática ou área, que enfatizaram fontes bibliográficas consultadas e autores mais citados, por exemplo, este artigo tem como foco as abordagens teóricas e metodológicas dos estudos de caso elaborados com destaque para os custos ambientais, podendo ter sido estudos de caso únicos ou múltiplos.

Além desta introdução, este artigo apresenta o referencial teórico que embasou os levantamentos e resultados, as conclusões e recomendações para pesquisas futuras.

\section{REFERENCIAL TEÓRICO}

\subsection{TIPOLOGIAS DE CLASSIFICAÇÃO DA PESQUISA E ESTUDO DE CASO NAS CIÊNCIAS SOCIAIS}

O objetivo desta seção é analisar a classificação dos estudos de caso nas ciências sociais, muitas vezes considerada uma estratégia de pesquisa, um procedimento metodológico ou um tipo de pesquisa, de acordo com a visão de cada autor. 
Para Demo (200I, p. I9), metodologia "É uma preocupação instrumental. Trata das formas de se fazer ciência. Cuida dos procedimentos, das ferramentas e dos caminhos". A metodologia relaciona-se com os objetivos e a finalidade da pesquisa.

De acordo com Bufoni (2002, p. I),

tendo explicitado o que fazer, isto é, os objetivos, problemas, e hipóteses de pesquisa, deve-se, então proceder ao detalhamento de como se pretende fazê-la, isto é, o método que será usado para se atingir tais objetivos.

A metodologia da pesquisa refere-se a uma etapa fundamental na elaboração de um trabalho científico, tendo em vista a necessidade de identificação das técnicas que serão adotadas para alcançar as respostas ao problema objeto de análise e investigação. Para isso, é preciso destacar os três grandes grupos que norteiam a classificação quanto aos objetivos da pesquisa, utilizados nas obras sobre metodologia sobre as mais diversas perspectivas: pesquisa exploratória, descritiva e explicativa.

A pesquisa exploratória é uma alternativa quando há pouco conhecimento acumulado sobre o tema a ser abordado, para conhecer mais sobre o assunto, construir teorias e formular hipóteses em trabalhos futuros.

Observa-se o caráter exploratório de um estudo quando as investigações sobre determinado assunto são preliminares na pesquisa acadêmica. Se esta pode ser uma oportunidade de iniciar a discussão sobre determinado assunto, há a desvantagem de acesso a poucas referências bibliográficas a respeito, o que não invalida proposta alguma que possa contribuir para o crescimento do debate em torno de temas considerados atuais e relevantes.

Assim, explorar um assunto significa reunir mais conhecimento e incorporar características inéditas, bem como buscar novas dimensões até então não conhecidas. O estudo exploratório apresenta-se como um primeiro passo no campo científico, a fim de possibilitar a realização de outros tipos de pesquisa acerca do mesmo tema, como a pesquisa descritiva e a explicativa (RAUPP; BEUREN, 2003, p. 8I).

A pesquisa exploratória é usada em casos nos quais é necessário definir o problema com maior precisão, identificar cursos relevantes de ação ou obter dados adicionais antes que se possa desenvolver uma abordagem. Entretanto, a finalidade que mais se encaixa nesse tipo de pesquisa é estabelecer prioridades para investigações posteriores (MALHOTRA, 200I; SELLTIZ et al., I965).

Quanto ao desenvolvimento de hipóteses, deve-se notar que, em muitas áreas do conhecimento científico, não existem hipóteses significativas. Por isso, é necessário fazer muitas pesquisas exploratórias antes de formulá-las. O tempo 
para a formulação das hipóteses varia com a natureza do problema e a amplitude do conhecimento anterior a respeito. A formulação e reformulação de problema de pesquisa representam um processo contínuo (SELLTIZ et al., I965).

A pesquisa descritiva descreve características de uma população ou fenômeno, além de estabelecer relações entre variáveis. Com um nível de profundidade maior do que a pesquisa exploratória, a pesquisa descritiva permite a identificação, análise e comparação de dados, porém sem a interferência do pesquisador.

A pesquisa explicativa, também conhecida como causal, busca explicar o porquê da ocorrência de determinado fenômeno, com maior aprofundamento sobre a causa e o efeito dele. De acordo com Hair Jr. et al. (2005, p. 89):

Os estudos causais testam se um evento causa outro evento ou não [...] uma relação causal significa que uma mudança em um evento provoca uma mudança correspondente em outro evento.

Com base numa análise bibliográfica das abordagens sobre tipologias utilizadas por diversos pesquisadores, Raupp e Beuren (2003) destacaram a falta de uniformidade existente ao verificarem que a pesquisa na área de ciências sociais pode ser classificada por tipologias sem agrupamentos, com agrupamentos e quanto a natureza, fins, meios, objetivos, procedimentos, objeto, fontes de informações e procedimentos de coleta.

No que diz respeito aos estudos de caso, para Yin (200I, p. 22), autor de uma das obras mais citadas sobre o tema: "os estudos de caso estão muito longe de ser apenas uma estratégia exploratória. Alguns dos melhores e mais famosos estudos de casos foram descritivos e explanatórios". Nessa direção, de acordo com Martins (2006, p. II): "segundo a abrangência e propósitos, um Estudo de Caso poderá ser exploratório, descritivo e experimental”, sendo este último raro nas ciências sociais aplicadas.

Tendo em vista os cuidados que devem ser tomados na elaboração de um estudo de caso, assim como as vantagens e desvantagens dessa estratégia de pesquisa, Yin (200I) apresentou importantes contribuições sobre os passos que devem ser seguidos na sua condução, como validação interna e externa do projeto, confiabilidade, cuidado na coleta de evidências e apresentação dos resultados, de forma que a validação e o rigor metodológico sejam reconhecidos pelos leitores de trabalhos que utilizem essa estratégia. Yin (200I) aponta também que a estratégia estudo de caso não é uma forma de coletar dados nem representa apenas a classificação de um estudo, pois observam-se os critérios para identificação da unidade de análise na pesquisa, que pode ser um indivíduo, uma empresa, uma pessoa, um setor ou situação específica. 
Yin (200I, p. 32) define estudo de caso como uma estratégia de pesquisa que "investiga um fenômeno contemporâneo dentro do seu contexto da vida real, especialmente quando os limites entre o fenômeno e o contexto não estão claramente definidos". Esse autor, considera essa estratégia, como outras de pesquisa, uma maneira de investigar um tópico empírico seguindo um conjunto de procedimentos pré-especificados.

Nas pesquisas conduzidas por Eisenhardt (I989, I99I, 2007), que são importantes referências na literatura acadêmica sobre estudo de caso, a autora apresentou alguns critérios que devem ser seguidos no processo de construção de teorias com base em estudos de caso. A autora enfatizou a testabilidade, validação empírica e relação com evidências como tópicos primordiais para a construção de teorias com base em estudos de caso.

Stake (2000) destacou a utilização de estudos de caso em pesquisas qualitativas. Para esse autor, o estudo de caso não é uma escolha metodológica, mas uma escolha sobre o que será estudado, e o propósito de um relatório de estudo de caso não é representar o mundo, mas representar o caso.

De acordo com Cooper e Schindler (2003, p. I20),

os estudos de caso colocam mais ênfase em uma análise contextual completa de poucos fatos ou condições e suas inter-relações. Um único estudo de caso bem planejado pode representar um desafio importante para uma teoria e simultaneamente ser a fonte de novas hipóteses e constructos.

No entendimento de Lazzarini (I997), a possibilidade de utilizar várias fontes de evidências é considerada uma das vantagens da pesquisa baseada em estudos de caso. Em contrapartida, o método não permite a extrapolação dos resultados nem a inferência estatística. A análise do estudo de caso permite extrair conclusões sugestivas, não capturáveis pelos métodos tradicionais.

Algumas críticas são muito comuns em relação aos estudos de caso, como a falta de rigor na condução da pesquisa, e quanto aos resultados destes, que não podem ser generalizados, limitando-se ao universo investigado. Entretanto, de acordo com Yin (200I, p. 29), "os estudos de caso, da mesma forma que os experimentos, são generalizáveis a proposições teóricas, e não a populações ou universos". Além disso, esse autor conclui que as discussões sobre a elaboração de estudo de caso único ou múltiplo também estão longe de um fim pacífico.

Bonoma (I985) propõe que não haja um "bom e perfeito" método de pesquisa, porque todos eles deverão refletir a limitação que um método intrinsecamente possui. 
Maior Filho (1984, p. I46-I47) defende o estudo de caso na pesquisa em administração:

O julgamento de uma pesquisa deveria ser sua relevância e não a abordagem usada [...] estudos de caso se constituem numa das abordagens mais eficazes e fecundas para a descoberta e posterior construção de modelos de processos sociais. A vantagem de estudos de caso é naturalmente ampliada quando vários instrumentos de coleta de dados primários e secundários são usados em conjunto.

Sobre essa perspectiva, Pozzebon e Freitas (1998) propuseram a sistematização das principais condições que podem proporcionar maior rigor científico à aplicação de um estudo de caso, estabelecendo um roteiro para sua aplicação.

De acordo com Joia (2004, p. I29): "Estudo de caso não pode ser classificado como uma metodologia de pesquisa qualitativa. Um mixer de evidências qualitativas e quantitativas usualmente é necessário para fundamentar o estudo de caso".

Verifica-se que a estratégia estudo de caso é utilizada com maior frequência nas pesquisas de caráter exploratório, tendo como diferencial para sua escolha as diversas técnicas de coleta de dados que podem ser aplicadas. Entretanto, o rigor metodológico deve ser considerado não somente na apresentação dos procedimentos adotados, mas também na análise e síntese dos resultados.

Antes de tudo, segundo Yin (200I), é preciso apresentar os passos que foram seguidos para a elaboração da investigação, o protocolo de pesquisa e a avaliação interna e externa do projeto. Outro aspecto relevante é a relação com o referencial da pesquisa, que deve demonstrar a ligação do trabalho de campo com os desenvolvimentos teóricos abordados nas pesquisas anteriores.

\subsection{ESTRATÉGIA ESTUDO DE CASO: PESQUISAS ANTERIORES}

O objetivo desta seção é apresentar os resultados de pesquisas que abordaram a análise qualitativa e/ou quantitativa da produção acadêmica sobre estudos de caso, de modo geral.

Bufoni (2002) avaliou a utilização de estudo de caso único, de uma amostra de 24 teses e dissertações de uma instituição de ensino superior (IES) brasileira. $\mathrm{O}$ autor utilizou as recomendações de Yin (200I) para estabelecer critérios de avaliação das pesquisas e identificou a falta de rigor na exposição dos elementos essenciais à credibilidade de um estudo de caso, principalmente por ter identificado a análise das evidências como a fase menos explorada pelos autores.

Gil, Licht e Oliva (2005) atribuem essas deficiências à falta de tradição no ensino de metodologia de pesquisa nos curso de graduação em administração. 
Por sua vez, Cardoso, Pereira e Guerreiro (2007) questionam a eficácia da aplicação do método na produção acadêmica de custos.

Gil, Licht e Oliva (2005) analisaram criticamente a utilização do estudo de caso como delineamento de pesquisa nos artigos apresentados no $26^{\circ}$ Encontro da Associação Nacional de Pós-Graduação e Pesquisa em Administração (Enanpad). Esses autores identificaram a utilização de poucas fontes de evidências na maioria das pesquisas, independentemente da área de enquadramento do estudo, além da falta de clareza nos procedimentos analíticos. De acordo com Gil, Licht e Oliva (2005, p. 51):

Note-se que contabilidade e controle gerencial é a sexta área com maior número de trabalhos apresentados e finanças, a sétima. Mas parte significativa desses trabalhos corresponde a análises de casos (não necessariamente estudos de caso) ou elaboração de propostas de ação. Isso indica que essas áreas apresentam notável vocação profissionalizante.

Com ênfase nas perspectivas epistemológicas positivista e interpretativista, De Vries (2004) analisou o conteúdo de uma amostra de 62 artigos publicados em 6 top journals europeus e americanos. Com foco na área de sistemas de informações gerenciais, esse autor utilizou os mesmos critérios estabelecidos por Benbasat, Goldstein e Mead (I987). Os resultados da pesquisa revelaram que os periódicos europeus tendem a ser mais abertos a enfoques menos tradicionais, pois publicam mais estudos interpretativistas do que os periódicos americanos. Entretanto, esses aspectos não diferem a aplicação da estratégia estudo de caso. Segundo De Vries (2004), embora os resultados tenham demonstrado algum "progresso", na comparação com pesquisas anteriores sobre a avaliação qualitativa de estudos de caso, como a explicitação das técnicas utilizadas para análise dos dados, apresentaram deficiências quanto ao banco de dados ou protocolo para elaboração do estudo, que ainda são raros.

Cepeda e Martin (2005) tiveram como objetivo propor um guia de referência para condução de estudos de casos, apoiados principalmente nas contribuições de Eisenhardt (1989) e Yin (200I). Cepeda e Martin (2005) utilizaram os seguintes critérios de avaliação: enfoque epistemológico das pesquisas, validade interna, validade do constructo, validade externa e confiabilidade. No âmbito do periódico Management Decision, esses autores analisaram qualitativamente o conteúdo de uma amostra de nove estudos de casos, com o objetivo de apresentar os aspectos positivos das categorias recomendadas para que as pesquisas qualitativas sejam utilizadas na construção de teorias. 
O estudo conduzido por Cardoso, Pereira e Guerreiro (2007) teve como objetivo traçar o perfil da pesquisa em custos no âmbito da temática de contabilidade e controle gerencial nos Enanpad de 1998 a 2003, considerando os aspectos qualitativos e quantitativos destes. Quanto aos estudos de caso analisados, esses autores constataram a falta de rigor metodológico e não identificaram as contribuições teóricas, além de não haver aprofundamento nas análises dos resultados.

No âmbito da tipologia de um estudo de caso, Grünbaum (2007) analisou criticamente as ambiguidades entre a especificação conceitual e a especificação da unidade de análise. Com base numa revisão bibliográfica sobre os conceitos elencados por autores clássicos da pesquisa nas ciências sociais, esse autor propôs alternativas que reúnem adaptações da tipologia proposta por Yin (200I).

Nos últimos anos, tem sido intensa a produção de pesquisas bibliométricas com foco na análise da produção acadêmica na área de administração e contabilidade, de um modo geral, ou em alguma temática específica. As abordagens mais frequentes são: teoria das organizações (MARIZ et al., 2004), administração (TINOCO, 2005), responsabilidade social das empresas (MORETTI; FIGUEIREDO, 2007), controle gerencial (LYRIO; BORBA; COSTA, 2007), finanças (CAMARGOS; COUTINHO; AMARAL, 2005) e evolução temporal da produção em contabilidade (MENDONÇA NETO; RICCIO; SAKATA, 2006).

Sobre os resultados de pesquisas que avaliaram a utilização da estratégia estudo de caso no Brasil, de acordo com Martins (2006, p. 8):

Muitos dos textos resultantes de pesquisas orientadas pela conveniente escolha de uma unidade mostram desenvolvimentos que não atentam para o necessário rigor científico, não conseguem ir além da exposição de enfadonhos relatórios, com excesso de detalhes totalmente dispensáveis, cujos resultados em nada surpreendem, pelo contrário, são plenamente conhecidos antes de serem apresentados pelos estudos.

Para Martins (2006, p. I8),

um grave defeito que se nota no desenvolvimento de diversos estudos de caso é a falta de uma plataforma teórica que aponte o que investigar, como demonstrar as proposições do estudo e que oriente a abordagem e aproximação com o fenômeno propriamente dito.

A pesquisa conduzida por Gallon et al. (2007) teve como objetivo classificar os artigos científicos da área ambiental publicados nos anais do Congresso 
USP de Controladoria e Contabilidade e no Encontro da Associação Nacional de Pós-Graduação e Pesquisa em Administração (Enanpad), como também em revistas de administração e contabilidade classificadas pela Coordenação de Aperfeiçoamento de Pessoal de Nível Superior (Capes) como "Nacional A" no período de 2000 a 2006 . Para um total de I86 artigos analisados, os autores identificaram quatro categorias para classificação - contabilidade ambiental, evidenciação ambiental, gestão ambiental e sustentabilidade - e observaram que há estreita conformidade e aproximação entre as categorias estabelecidas na pesquisa.

Teodósio et al. (2007) analisaram as teses e dissertações das linhas de pesquisa de ética nos negócios e gestão do meio ambiente, de uma IES estabelecida em São Paulo, no período de I986 a 2004. Esses autores classificaram os trabalhos quanto aos procedimentos metodológicos adotados e constataram a forte presença de estudos de caso nas pesquisas sobre meio ambiente.

Mathews (I997) revisou a literatura acadêmica internacional sobre o gerenciamento de custos e verificou que a pesquisa sobre o tema, nos últimos 25 anos, produziu poucas informações sobre o envolvimento de custos e técnicas gerenciais. O autor concluiu que a maior parte das contribuições é retida internamente nas empresas, para uso dos gerentes e não para divulgação entre os demais stakeholders.

A revisão bibliográfica de pesquisas anteriores que enfatizaram a estratégia estudo de caso, tanto na área de administração como em contabilidade, apresentou resultados semelhantes, ou seja, falta de rigor na condução dos estudos e, sobretudo, deficiências nas análises dos resultados alcançados com a investigação.

As razões atribuídas referem-se ao pouco destaque no ensino de metodologia de pesquisa nos cursos de graduação, além da trajetória, experiência ou vocação dos alunos, mais voltados para o campo profissional do que acadêmico.

Considera-se importante o destaque desses resultados e principalmente a proposição de soluções para que o maior rigor na condução de pesquisas seja estabelecido, proporcionando maior credibilidade na análise dos resultados. Nessa direção e utilizando pesquisa de sua própria autoria como exemplo, Weerd-Nederhof (200I) contribuiu com a discussão metodológica da pesquisa qualitativa ao sugerir uma estrutura para elaboração de estudo de caso, com os passos a serem seguidos em todas as etapas.

Outro exemplo recente foi proposto por Martins (2006) que também apresentou recomendações importantes quanto aos passos que devem ser seguidos para que a estratégia estudo de caso seja utilizada de modo que os resultados da pesquisa sejam consistentes, além de esclarecer o que é e o que não é um estudo 
de caso, tendo em vista a percepção do autor quanto às deficiências recorrentes nas pesquisas que avaliaram epistemologias aplicadas em trabalhos que utilizaram a estratégia.

\section{PROCEDIMENTOS METOdOLÓGICOS}

Esta seção apresenta os passos para elaboração da pesquisa, classificada como descritiva. Quanto aos procedimentos adotados para a coleta de dados, utilizaram-se a pesquisa bibliográfica, que apoiou a revisão da produção acadêmica sobre o tema deste artigo, e a criação de categorias para a análise de conteúdo, técnica importante para a investigação dos aspectos teóricos e metodológicos dos estudos de caso.

\subsection{PERFIL DOS TRABALHOS SELECIONADOS}

Efetuou-se, inicialmente, uma busca nas bibliotecas das IESs que oferecem programas de pós-graduação stricto sensu, de acordo com a relação destes, que são recomendados e reconhecidos pela Capes. Em seguida, foram identificadas as teses e dissertações defendidas para posterior localização, acesso, seleção e análises.

Identificaram-se as dissertações e teses em que os autores utilizaram a estratégia estudo de caso, único ou múltiplo, com ênfase nos custos ambientais. O critério utilizado foi a acessibilidade das pesquisas, independentemente da IES e da classificação atribuída pela Capes.

Verificou-se que há uma grande produção sobre custos ambientais em linhas de pesquisas de programas de pós-graduação dos cursos de engenharia oferecidos por várias IESs, com destaque para a área de engenharia de produção. Por tratar-se de trabalhos que se enquadram no tema desta pesquisa, também foram incluídos na análise.

As teses e dissertações que tiveram o estudo de caso como estratégia de pesquisa sobre custos ambientais foram selecionadas a partir do ano que estavam disponíveis na biblioteca virtual de cada IES. Os trabalhos selecionados se referem aos cursos de mestrado e ou doutorado dos cursos de administração, ciências contábeis e engenharia. Nesse levantamento, obteve-se o resultado apresentado a seguir. 


\section{QUADRO I}

TESES E DISSERTAÇÖES QUE UTILIZARAM A ESTRATÉGIA ESTUDO DE CASO SOBRE CUSTOS AMBIENTAIS

\begin{tabular}{lccccccccc}
\hline TESES E DISSERTAÇÕES & 1997 & 2001 & 2002 & 2003 & 2004 & 2005 & 2006 & 2007 & TOTAL \\
\hline ADMINISTRAÇÃO & & & & 1 & 1 & 1 & 2 & 1 & $\mathbf{6}$ \\
CIÊNCIAS CONTÁBEIS & & & 2 & 3 & & 1 & 2 & 1 & $\mathbf{9}$ \\
\hline ENGENHARIA & 2 & 4 & 3 & 5 & 2 & 1 & 1 & 1 & $\mathbf{1 9}$ \\
\hline TOTAL & $\mathbf{2}$ & $\mathbf{4}$ & $\mathbf{5}$ & $\mathbf{9}$ & $\mathbf{3}$ & $\mathbf{3}$ & $\mathbf{5}$ & $\mathbf{3}$ & $\mathbf{3 4}$ \\
\hline
\end{tabular}

Fonte: Elaborado pela autora.

Observação: O primeiro trabalho sobre o tema desta pesquisa é de I997. Foram omitidos os períodos em que não foram localizados trabalhos relacionados com o tema da investigação.

\subsection{ANÁLISE DE CONTEÚdO}

A análise de conteúdo é uma técnica que permite a classificação de frases, palavras ou partes de textos para posterior quantificação e verificação da frequência, das características e particularidades, de acordo com o tema em estudo. Essa técnica é definida por Bardin (2004, p. 27-28) como

um conjunto de técnicas de análise das comunicações, visando a obter, por procedimentos sistemáticos e objetivos de descrição do conteúdo das mensagens, indicadores quantitativos ou não, que permitam a inferência de conhecimentos relativos às condições de produção/recepção (variáveis inferidas) das mensagens.

Os campos de aplicação da análise de conteúdo são amplos, e, neste trabalho, adotaram-se as três etapas para sua utilização, de acordo com as recomendações de Bardin (2004):

- $\quad$ Primeira etapa (pré-análise): leitura das teses e dissertações para preparação do material.

- Segunda etapa: análise do material selecionado, codificação e escolha das regras de contagem das unidades de registro para posterior categorização.

- Terceira etapa: categorias preestabelecidas para então buscar a interpretação e os sentidos daquilo que os dados revelam. 
Freitas, Cunha Júnior e Moscarola (I997, p. 6) argumentam que "as categorias devem ter origem no documento objeto de análise ou em certo conhecimento geral da área do conhecimento ou da atividade no qual se insere". As unidades de análise escolhidas foram as sentenças que qualificavam o estudo de caso, conforme cada categoria estabelecida, para posterior codificação e quantificação. Os trabalhos acadêmicos foram sistematizados para uma avaliação qualitativa dos estudos disponibilizados com ênfase em custos ambientais, cujos resultados são apresentados na próxima seção.

A revisão bibliográfica sobre o tema desta pesquisa e a leitura inicial das teses e dissertações contribuíram para a criação das categorias aqui apresentadas, com base na percepção do que é um estudo de caso, o que deve conter, como se classifica, o conteúdo e a forma de apresentação da metodologia, além da relação dessa estrutura com os resultados e as conclusões revelados nas pesquisas.

\section{QUADRO 2}

\section{CATEGORIAS PARA ANÁLISE DE CONTEÚDO DAS} TESES E DISSERTAÇÕES SELECIONADAS

\begin{tabular}{|c|c|c|c|}
\hline & \multicolumn{2}{|l|}{ CLASSIFICAÇÃO DO ESTUDO } & $\begin{array}{l}\text { TÉCNICAS UTILIZADAS PARA COLETA DE } \\
\text { DADOS }\end{array}$ \\
\hline 1 & Pesquisa qualitativa. & 14 & Entrevistas. \\
\hline 2 & Pesquisa quantitativa. & 15 & Aplicação de questionário. \\
\hline 3 & Abordagem qualitativa e quantitativa. & 16 & Pesquisa documental. \\
\hline 4 & Proposição e aplicação de modelos. & 17 & Observação participante. \\
\hline \multirow[t]{3}{*}{5} & Descrição de algum fenômeno/evento. & 18 & Análise de conteúdo. \\
\hline & & 19 & Artefatos físicos e lógicos. \\
\hline & CONSIDERAÇÕES GERAIS & & QUANTO AOS RESULTADOS OBTIDOS \\
\hline 6 & $\begin{array}{l}\text { Trata-se de um estudo de caso, mas está } \\
\text { implícito. }\end{array}$ & 20 & Comparação com a teoria referenciada. \\
\hline 7 & $\begin{array}{l}\text { É explicitado e justificado o estudo de } \\
\text { caso. }\end{array}$ & 21 & Benefícios para a empresa. \\
\hline 8 & $\begin{array}{l}\text { Trata-se de estudo de caso classificado } \\
\text { de outra forma. }\end{array}$ & 22 & $\begin{array}{l}\text { Resultados da aplicação qualitativa/ } \\
\text { quantitativa. }\end{array}$ \\
\hline
\end{tabular}




\section{QUADRO 2 (CONTINUAÇÃO)}

\section{CATEGORIAS PARA ANÁLISE DE CONTEÚDO DAS} TESES E DISSERTAÇÕES SELECIONADAS

\begin{tabular}{|c|c|c|c|}
\hline & CLASSIFICAÇÃO DO ESTUDO & & $\begin{array}{l}\text { TÉCNICAS UTILIZADAS PARA COLETA DE } \\
\text { DADOS }\end{array}$ \\
\hline 9 & É explicitado, mas não justificado. & 23 & $\begin{array}{l}\text { Reporte às contribuições teóricas e } \\
\text { práticas do estudo. }\end{array}$ \\
\hline & PROCEDIMENTOS METODOLÓGICOS & & AVALIAÇÃO DOS ESTUDOS DE CASO \\
\hline 10 & Referências teóricas. & 24 & $\begin{array}{l}\text { Validade interna (diversidade de } \\
\text { técnicas). }\end{array}$ \\
\hline 11 & $\begin{array}{l}\text { Não há referências teóricas sobre estudo } \\
\text { de caso. }\end{array}$ & 25 & $\begin{array}{l}\text { Validade do constructo (múltiplas fontes } \\
\text { de evidências, encadeamento das } \\
\text { evidências, revisão do relatório efetuada } \\
\text { por informante-chave). }\end{array}$ \\
\hline 12 & $\begin{array}{l}\text { Discrimina os passos seguidos para } \\
\text { elaboração do estudo de caso } \\
\text { (protocolo). }\end{array}$ & 26 & Validade externa. \\
\hline 13 & A unidade de análise é discriminada. & 27 & Confiabilidade. \\
\hline
\end{tabular}

Fonte: Elaborado pela autora.

\section{RESULTADOS E DISCUSSÕES}

Foi analisado o total de 34 trabalhos acadêmicos, ou seja, 5 teses e 29 dissertações. Tendo em vista as diversas categorias estabelecidas para análise neste artigo, os resultados foram apresentados com base em cada temática.

\subsection{ESTUDOS DE CASOS: QUANTO À CLASSIFICAÇÃO}

Teses e dissertações são trabalhos que exigem maior dedicação, tempo, esforço e atenção ao rigor metodológico e, por isso, devem apresentar um referencial teórico amplo, além de adequado conteúdo qualitativo e quantitativo das análises e dos procedimentos metodológicos adotados.

Considerou-se nessa categoria a classificação dos estudos de caso quanto ao tipo de pesquisa e verificou-se que $50 \%$ dos trabalhos são do tipo qualitativo. Não foi localizada nenhuma tese ou dissertação que pudesse ser classificada como 
quantitativa. A abordagem qualitativa e quantitativa foi utilizada em $23,5 \%$ das pesquisas. Em I2,5\% dos trabalhos, privilegiaram-se a proposição e aplicação de modelos para mensuração e registro dos custos ambientais. Em I4,O\% das teses e dissertações, os autores propuseram a descrição de algum fenômeno ou evento relacionado com custos ambientais e verificaram como este ocorre em determinada organização.

Esses resultados confirmam verificações anteriores com foco em outras áreas, em que os autores destacaram a pouca frequência de pesquisas quantitativas que tenham utilizado a estratégia estudo de caso. A inclusão do item "Descrição de algum fenômeno/evento" deveu-se à grande ênfase deste objetivo comumente associado a um estudo de caso, mas não necessariamente com os procedimentos adotados na coleta de dados que qualifiquem a pesquisa como tal. O Gráfico I apresenta os resultados para essa categoria.

\section{GRÁFICO I}

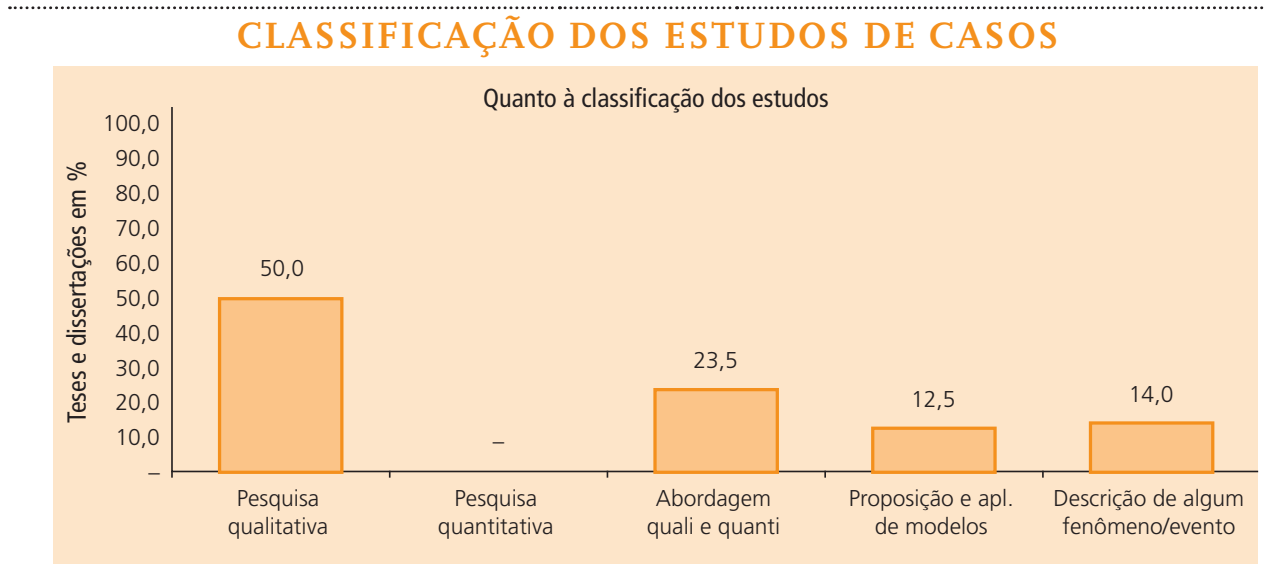

Fonte: Elaborado pela autora.

\subsection{CONSIDERAÇÕES GERAIS SOBRE AS PESQUISAS}

Examinou-se como os autores explicitaram a caracterização da pesquisa. Das pesquisas analisadas, I7,6\% referem-se a estudos de caso, mas esse dado está implícito, pois os autores classificaram esses trabalhos de outra forma em 6,5\% das teses e dissertações. Outro ponto que merece destaque: em $45,3 \%$ das pesquisas, os autores explicitaram tratar-se de estudo de caso, além de justificarem a utilização da estratégia. No entanto, em 30,6\% os autores explicitaram tratar-se de um estudo de caso, embora não tenham sido justificados. O Gráfico 2 apresenta os resultados para essa categoria. 


\section{GRÁFICO 2}

\section{CONSIDERAÇÕES GERAIS SOBRE AS PESQUISAS}

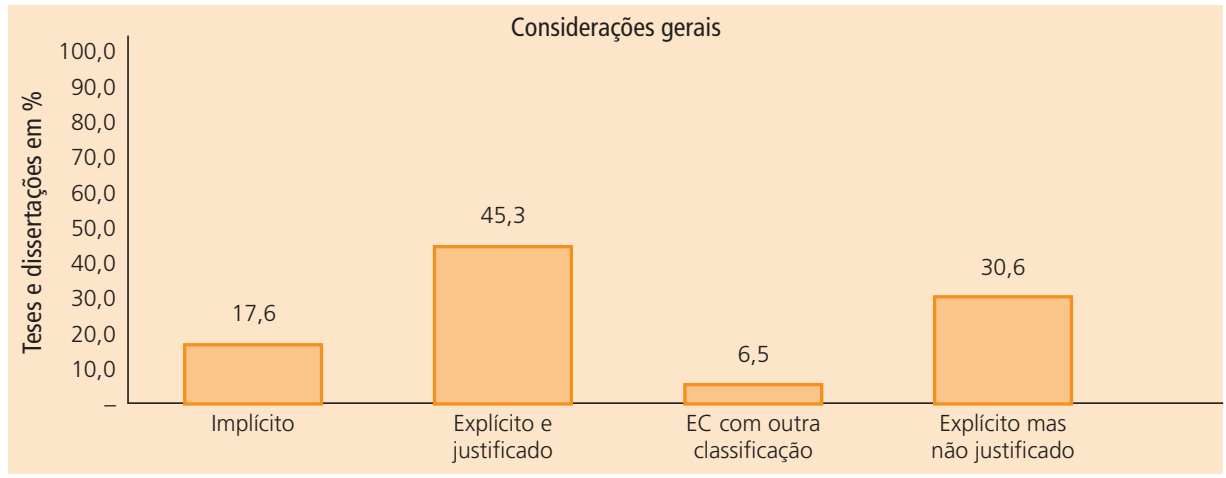

Fonte: Elaborado pela autora.

\subsection{PROCEDIMENTOS METODOLÓGICOS ADOTADOS NAS PESQUISAS}

Verificou-se como foram apresentados os procedimentos metodológicos adotados pelos autores, que enfatizassem as vantagens e desvantagens da utilização da estratégia estudo de caso. Em 48,4\% das pesquisas, os autores apresentaram o referencial teórico a respeito das técnicas utilizadas. Entretanto, em 34,2\% dos trabalhos, não há referencial teórico, ou seja, não foi apresentado um embasamento que justificasse as técnicas adotadas. Das pesquisas analisadas, II, $8 \%$ apresentaram os passos seguidos para elaboração do estudo de caso, mas somente 5,6\% explicitaram a unidade de análise da pesquisa, como mostra o Gráfico 3.

\section{GRÁFICO 3}

\section{PROCEDIMENTOS METODOLÓGICOS ADOTADOS NAS PESQUISAS}

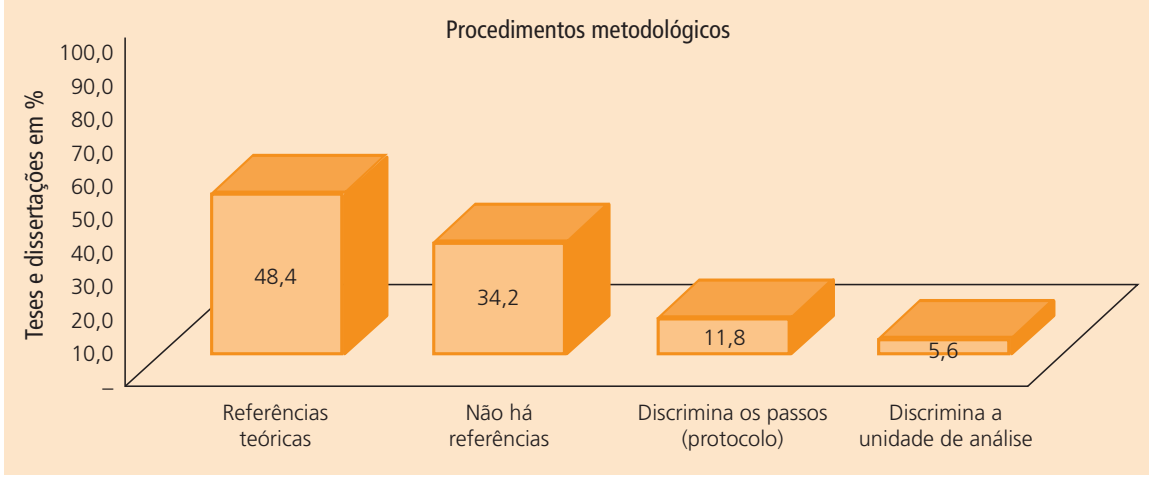

Fonte: Elaborado pela autora. 


\subsection{TÉCNICAS UTILIZADAS PARA COLETA DE DADOS}

Como visto no item "Referencial teórico" deste trabalho, uma das vantagens da estratégia estudo de caso é a diversidade de técnicas que podem ser utilizadas para coleta de dados, o que é considerado positivo para a validação do método. No conjunto investigado, observou-se que os métodos de coleta de dados que mais se destacaram na pesquisa sobre estudo de caso foram pesquisa documental, entrevistas e aplicação de questionários. Pesquisa observação-participante, análise de conteúdo e artefatos físicos e lógicos foram as técnicas menos utilizadas, como mostra o Gráfico 4.

\section{GRÁFICO 4}

\section{TÉCNICAS UTILIZADAS PARA COLETA DE DADOS}

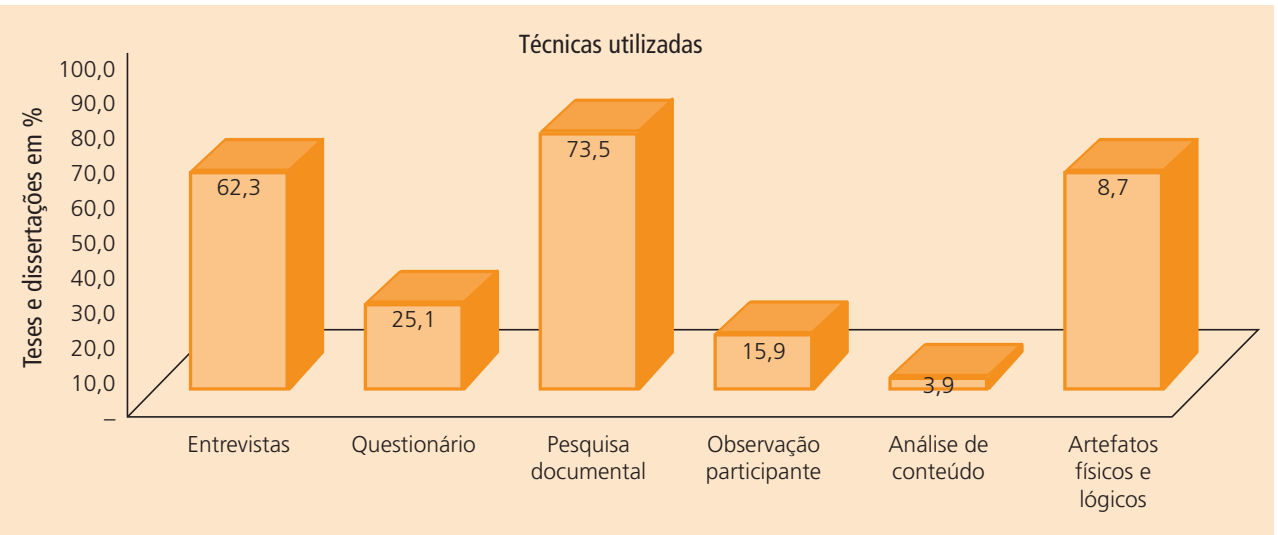

Fonte: Elaborado pela autora.

\subsection{QUANTO AOS RESULTADOS DAS PESQUISAS}

Observou-se que há grande preocupação com os seguintes aspectos: apresentação dos benefícios gerados pela aplicação de determinado método de sistema de custos ambientais, demonstração, qualitativa ou quantitativa, dos resultados gerados pelos controles e identificação dos custos ambientais. O Gráfico 5 apresenta os resultados para essa categoria. 


\section{GRÁFICO 5}

\section{RESULTADOS DAS PESQUISAS ANALISADAS}

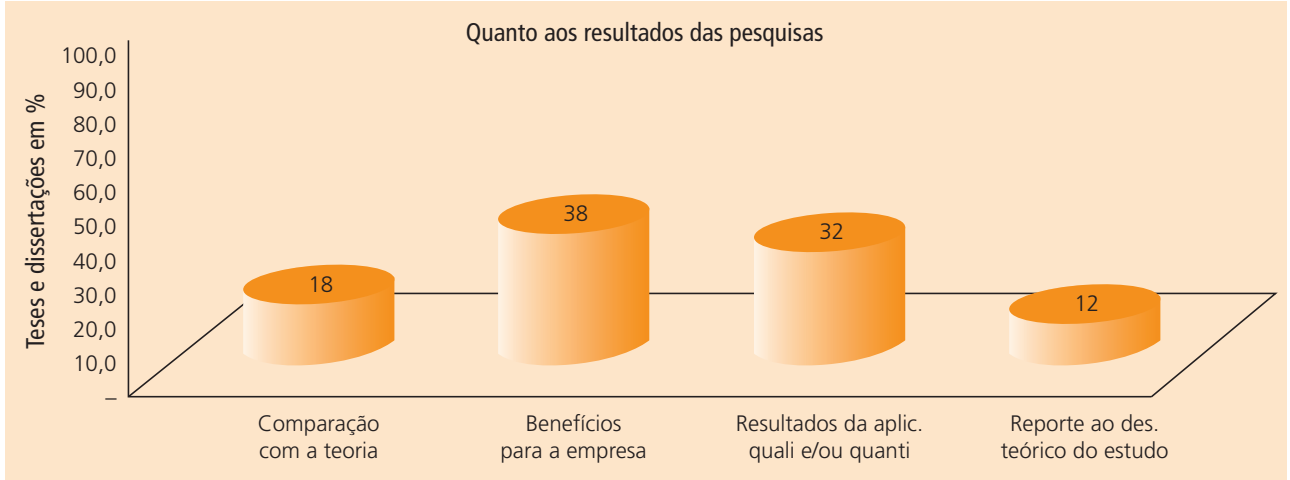

Fonte: Elaborado pela autora.

\subsection{AVALIAÇÃO DOS ESTUdOS DE CASOS}

Essa categoria teve por objetivo avaliar os estudos de caso sobre custos ambientais com base nas proposições de Yin (200I) para validação das pesquisas. Foi possível aplicar os critérios somente para validade interna, tendo em vista que se identificou a diversidade de técnicas utilizadas, embora estas ainda não sejam significativamente exploradas.

Não foi possível verificar a validade do constructo, porque os autores não explicitaram o protocolo de pesquisa, de modo que esclarecesse as fontes de evidências para justificação dos resultados obtidos. Os quesitos validade externa e confiabilidade também foram prejudicados, por não haver referências nas pesquisas que possibilitassem essas verificações.

Constatou-se que os resultados dos estudos de caso, em sua maioria, não relacionaram a aplicação prática proposta com os desenvolvimentos teóricos anteriores, os quais, em muitos trabalhos, foram amplamente destacados na revisão bibliográfica.

\section{CONSIDERAÇÕES FINAIS}

O objetivo deste trabalho foi analisar os estudos de caso que abordaram a identificação e mensuração de custos ambientais. Apresentou-se, inicialmente, um referencial teórico sobre a definição e os pontos fortes e fracos da estratégia estudo de caso. Em seguida, abordou-se a revisão bibliográfica de pesquisas ante- 
riores que analisaram qualitativa e quantitativamente a produção de trabalhos apresentados em congressos, assim como teses e dissertações de diversas áreas temáticas.

Verificou-se que os resultados de pesquisas anteriores apontaram muitas deficiências na utilização dessa estratégia, relacionadas principalmente com a falta de rigor metodológico. Observou-se também pouca exploração dos resultados obtidos com o estudo e da relação deste com o referencial teórico abordado na pesquisa.

Embora muito utilizada nas ciências sociais, essa estratégia exige maior empenho do pesquisador, para que o estudo de caso inspire a confiabilidade do método, das técnicas utilizadas e dos resultados obtidos.

Foram analisadas 34 teses e dissertações que abordaram a estratégia estudo de caso sobre custos ambientais, dos programas de pós-graduação de instituições de ensino superior reconhecidas pela Capes. Destes trabalhos, 29 se referiram a dissertações de mestrado e 5 a teses de doutorado, dos cursos de administração, ciências contábeis e engenharia. A análise de conteúdo das pesquisas revelou a predominância de estudos de caso com ênfase na abordagem qualitativa, o que não sugere vantagem ou desvantagem, tendo em vista que os estudos de caso raramente são utilizados em pesquisas quantitativas.

A proposição e aplicação de modelos para mensuração e registro de custos ambientais é um tema presente em I2,5\% das pesquisas analisadas, sugerindo, assim, um campo amplo a ser explorado, inclusive com a utilização da estratégia estudo de caso, unicamente ou em conjunto com outras abordagens.

Em I4, O\% das teses e dissertações, os autores propuseram a descrição de algum fenômeno ou evento relacionado com custos ambientais. Os resultados desse tipo de abordagem foram enfraquecidos pela falta de relacionamento dos achados com o referencial teórico da pesquisa em si e com os procedimentos metodológicos adotados no estudo de caso.

Outro resultado importante obtido com a análise de conteúdo refere-se ao fato de que, em $45,3 \%$ das pesquisas, os autores explicitaram tratar-se de um estudo de caso, além de justificarem a utilização da estratégia. Porém, em 30,6\% dos trabalhos, os autores explicitaram que a pesquisa tratava-se de um estudo de caso, o que não foi justificado.

A verificação dos procedimentos metodológicos adotados pelos autores revelou que, em $48,4 \%$ das pesquisas, os autores apresentaram o referencial teórico a respeito das técnicas utilizadas. Porém, em $34,2 \%$ dos trabalhos, não foi apresentado um embasamento que justificasse as técnicas adotadas.

Observou-se que os métodos de coleta de dados mais utilizados na pesquisa sobre estudo de caso foram: entrevistas, pesquisa documental e aplicação de questionário. A utilização de diversas técnicas é uma das formas de validação 
do estudo de caso, e, embora estas tenham sido muito utilizadas, a verificação demonstrou que outras técnicas, como análise de conteúdo e observação participante, poderiam ter sido mais exploradas.

Verificou-se que há grande preocupação com os seguintes aspectos: apresentação dos benefícios gerados pela aplicação de determinado método de sistema de custos ambientais, demonstração, qualitativa ou quantitativa, dos benefícios gerados pelos controles e identificação destes.

As análises revelaram que esse tipo de conclusão não remete aos objetivos propostos no estudo de caso, além de não confirmar ou refutar a teoria referenciada na pesquisa.

Não foi plenamente aplicável a validação das pesquisas, porque os autores não explicitaram o protocolo, de modo que esclarecesse as fontes de evidências coletadas para justificação dos resultados obtidos. Os quesitos validade externa e confiabilidade também não foram aplicáveis.

Observou-se que os resultados dos estudos de caso, em sua maioria, não relacionaram a aplicação prática que foi proposta com os desenvolvimentos teóricos anteriores, os quais, em muitos trabalhos, foram amplamente destacados na revisão bibliográfica.

Recomendam-se, para futuros estudos, a ampliação da amostra para outras áreas de pesquisa e a aplicação da análise de conteúdo com ênfase no tema deste trabalho em artigos publicados em periódicos, como também apresentados em congressos, encontros, seminários e simpósios.

\section{REFERÊNCIAS}

BARDIN, L. Análise de conteúdo. Tradução Luis A. Reto e Augusto Pinheiro. Lisboa: Edições 70, LDA, 2004 .

BENBASAT, I.; GOLDSTEIN, D. K.; MEAD, M. The case research strategy in studies of information systems. MIS Quartely, v. II, n. 3, p. 369-386, I987.

BONOMA, T. V. Case research in marketing: opportunities, problems and a process. Journal of Marketing Research, Chicago, v. XXII, p. I99-208, May I985.

BUFONI, A. L. O rigor na exposição dos estudos de caso simples: um teste empírico em uma universidade brasileira. In: ENCONTRO DA ASSOCIAÇÃO NACIONAL DE PÓS-GRADUAÇÃO E PESQUISA EM ADMINISTRAÇÃO, 26., 2002, Atibaia. Anais... Atibaia: Anpad, 2002.

CAMARGOS, M. A.; COUTINHO, E. S.; AMARAL, H. F. O perfil da área de finanças do Enanpad: um levantamento da produção científica e de suas tendências entre 2000-2004. In: ENCONTRO DA ASSOCIAÇÃO NACIONAL DE PÓS-GRADUAÇÃO E PESQUISA EM ADMINISTRAÇÃO, 29., 2005, Brasília. Anais... Brasília: Anpad, 2005. I CD-ROM.

CARDOSO, R. L.; PEREIRA, C. A.; GUERREIRO, R. Perfil das pesquisas em contabilidade de custos apresentadas no Enanpad no período de I998 a 2003. RAC, v. II, n. 3, p. I77-I98, jul./set. 2007 . 
CEPEDA, G.; MARTIN, D. A review of case studies publishing in Management Decision 20032004. Management Decision, v. 43, n. 6, p. 85I-876, 2005.

COOPER, D. R.; SCHINDLER, P. S. Métodos de pesquisa em administração. Porto Alegre: Bookman, 2003.

COORDERNAÇÃO DE APERFEIÇOAMENTO DE PESSOAL DE NÍVEL SUPERIOR (Capes). Disponível em: <www.capes.gov.br>. Acesso em: I3 jul. 2007.

DE VRIES, E. J. Epistemology and methodology in case research: a comparison between European and Americans is journals. American IS Journals, Primavera Working Paper, 2004. Disponível em: <is2.lse.ac.uk/asp/aspecis/20050II3.pdf>. Acesso em: 25 nov. 2007.

DEMO, P. Saber pensar. 2. ed. São Paulo: Cortez, 200I.

EISENHARDT, K. M. Building theories from case study research. The Academy of Management Review, v. I4, n. 4, p. 532-550, Oct. I989.

Better stories and better constructs: the case for rigor and comparative logic. The Academy of Management Review, v. I6, n. 3, p. 620-627, July I991.

Theory building from cases: opportunities and challenges. The Academy of Management Review, v. 50, n. I, p. 25-32, 2007.

FREITAS, H. M. R.; CUNHA JÚNIOR, M. V. M.; MOSCAROLA, J. Aplicação de sistema de software para auxílio na análise de conteúdo. Revista de Administração da Universidade de São Paulo, São Paulo, v. 32, n. 3, p. 97-I09, jul./set. I997.

GALLON, A. V. et al.. Produção científica e perspectivas teóricas da área ambiental: um levantamento a partir de artigos publicados em congressos e periódicos nacionais da área de contabilidade e administração. In: CONGRESSO USP DE CONTROLADORIA E CONTABILIDADE, 7., 2007, São Paulo. Anais... São Paulo: FEA, 2007. I CD-ROM.

GIL, A. C.; LICHT, R. H. G.; OLIVA, E. C. A utilização de estudos de caso na pesquisa em administração. BASE - Revista de Administração e Contabilidade da Unisinos, v. 2, n. I, p. 47-56, jan./abr. 2005.

GRÜNBAUM, N. N. Identification of ambiguity in the case study research typology: case study research typology: what is a unit of analysis? Qualitative Market Research: An International Journal, v. IO, n. I, p. 78-97, 2007.

HAIR JR., J. F. et al. Fundamentos de métodos de pesquisa em administração. Tradução: Lene Belon Ribeiro. Porto Alegre: Bookman, 2005.

JOIA, L. A. Geração de modelos teóricos a partir de estudos de casos múltiplos: da teoria à prática. In: VIEIRA, M. M. F.; ZONAIN, D. M. (Org.). Pesquisa qualitativa em administração. Rio de Janeiro: FGV, 2004 .

LAZZARINI, S. G. Estudos de caso para fins de pesquisa: aplicabilidade e limitações do método. In: FARINA, E. M. M. Q. et al. (Coord.). Estudos de caso em agribusiness. São Paulo: Pioneira, I997. p. 9-23.

LYRIO, M. V. L.; BORBA, J. A.; COSTA, J. M. Controle gerencial: delineamento do perfil metodológico de uma amostragem de publicações acadêmicas nas áreas de administração e contabilidade de 2000 a 2004. BASE - Revista de Administração e Contabilidade da Unisinos, v. 4, n. 2, p. I26-ı36, maio/ago. 2007.

MAIOR FILHO, J. S. Pesquisa em administração: em defesa do estudo de caso. Revista de Administração de Empresas, Rio de Janeiro, v. 24, n. 4, p. I46-I49, I984. 
MALHOTRA, N. K. Pesquisa de marketing: uma orientação aplicada. 3. ed. Porto Alegre: Bookman, 2OOI.

MARIZ, L. A. et al. O reinado dos estudos de caso em teoria das organizações: imprecisões e alternativas. In: Encontro de Estudos Organizacionais (Eneo), 3., 2004, Atibaia. Anais... Atibaia: Anpad, 2004. I CD-ROM.

MARTINS, G. A. Estudo de caso: uma estratégia de pesquisa. São Paulo: Atlas, 2006.

MATHEWS, M. R.Twenty-five years of social and environmental accounting research. Is there a silver jubilee to celebrate? Accounting, Auditing \& Accountability Journal, v. Io n. 4, p. 48I-53I, I997.

MENDONÇA NETO, O. R.; RICCIO, E. L.; SAKATA, M. C. G. Paradigmas da pesquisa em contabilidade no Brasil: Enanpad: I98I-2005. In: ENCONTRO DA ASSOCIAÇÃO NACIONAL DE PÓS-GRADUAÇÃO E PESQUISA EM ADMINISTRAÇÃO, 30., 2006, Salvador. Anais... Salvador: Anpad, 2006. I CD-ROM.

MORETTI, S. L. A.; FIGUEIREDO, J. C. B. Análise bibliométrica da produção sobre responsabilidade social das empresas no Enanpad: evidências de um discurso monológico. In: ENCONTRO DA ASSOCIAÇÃO NACIONAL DE PÓS-GRADUAÇÃO E PESQUISA EM ADMINISTRAÇÃO, 3I., 2007, Rio de Janeiro. Anais... Rio de Janeiro: Anpad, 2007. I CD-ROM.

POZZEBON; M.; FREITAS, H. M. R. Pela aplicabilidade com um maior rigor científico dos estudos de caso em sistemas de informação. RAC, v. 2, n. 2, p. I43-168, maio/ago. I998.

RAUPP, F. M.; BEUREN, I. M. Metodologia da pesquisa aplicável às ciências sociais. In: BEUREN, I. M. (Org.). Como elaborar trabalhos monográficos em contabilidade: teoria e prática. São Paulo: Atlas, 2003.

SANTOS, A. R. Metodologia científica: a construção do conhecimento. Rio de Janeiro: DP\&A, I999.

SELLTIZ, C. et al. Métodos de pesquisa das relações sociais. São Paulo: Herder, I965.

STAKE, R. E. Qualitative case studies. In: DENZIN, N. K.; LINCON, Y. S. (Ed.). Handbook of qualitative research. California: Sage, 2000.

TEODÓSIO, A. S. S. et al.. Produção de conhecimento e de teses e dissertações em administração: uma análise das linhas de pesquisa ética nos negócios e gestão do meio ambiente. In: ENCONTRO DA ASSOCIAÇÃO NACIONAL DE PÓS-GRADUAÇÃO E PESQUISA EM ADMINISTRAÇÃO, 31., 2007, Rio de Janeiro. Anais... Rio de Janeiro: Anpad, 2007. I CD-ROM.

TINOCO, T. A produção científica de administração no Brasil I997-2002: uma perspectiva bibliométrica. 2005. 54 f. Dissertação (Mestrado em Administração de Empresas)-Fundação Getulio Vargas, São Paulo, 2005.

WEERD-NEDERHOF, P. C. Qualitative case study research. The case of a PhD research project on organizing and managing new product development systems. Management Decision, v. 29, n. 7, p. 5I3-538, 200 I.

YIN, R.K. Estudo de caso: planejamento e métodos. Tradução: Daniel Grassi. 2. ed. Porto Alegre: Bookman, 200I. 